\title{
Application of IP-based Analog Platforms in the design of Neuromimetic Integrated Circuits
}

\author{
Timothée Levi, Noëlle Lewis Member IEEE, Jean Tomas, and Sylvie Renaud Member IEEE
}

\section{Affiliation:}

T. Levi is with the IMS laboratory, UMR CNRS 5218, University of Bordeaux, Talence, FR 33400, FRANCE. (phone: +33 54000 2773; fax: +3355637 1545; e-mail: timothee.levi@ims-bordeaux.fr).

N. Lewis is with the IMS laboratory, UMR CNRS 5218, University of Bordeaux, Talence, FR 33400, FRANCE (e-mail: noelle.lewis@ims-bordeaux.fr).

J. Tomas is with IMS laboratory, UMR CNRS 5218, University of Bordeaux, Talence, FR 33400, FRANCE (e-mail: jean.tomas@ims-bordeaux.fr).

S. Renaud is with the IMS laboratory, UMR CNRS 5218, University of Bordeaux, Talence, FR 33400, FRANCE (e-mail: sylvie.renaud@ims-bordeaux.fr).

No preliminary versions have been published

Final version of the paper (after 2 revisions)

Manuscript received July 28, 2011, first revision on October 30, 2011, second revised and re-submitted March 12, 2012, final version June 1, 2012.

T. Lévi is with the IMS laboratory, UMR CNRS 5218, University of Bordeaux, Talence, FR 33400, FRANCE. (phone: +33 54000 2773; fax: +33 55637 1545; e-mail: timothee.levi@ims-bordeaux.fr).

N. Lewis is with the IMS laboratory, UMR CNRS 5218, University of Bordeaux, Talence, FR 33400, FRANCE (e-mail: noelle.lewis@imsbordeaux.fr).

J. Tomas is with the IMS laboratory, UMR CNRS 5218, University of Bordeaux, Talence, FR 33400, FRANCE (e-mail: jean.tomas@imsbordeaux.fr).

S. Renaud is with the IMS laboratory, UMR CNRS 5218, University of Bordeaux, Talence, FR 33400, FRANCE (e-mail: sylvie.renaud@imsbordeaux.fr). 


\title{
Application of IP-based Analog Platforms in the design of Neuromimetic Integrated Circuits
}

\author{
Timothée Levi, Noëlle Lewis Member IEEE, Jean Tomas, and Sylvie Renaud Member IEEE
}

\begin{abstract}
Re-use methodologies are now widely used to design digital circuits. They are based on the concept of Intellectual Property (IP), or virtual block of computing, characterized by a behavioral model, synthesizable or not. The design re-use for analog integrated systems is much less natural and less standardized. This paper addresses the issue of an analog design flow based on re-use, focusing on those three key questions: the formal content of the IP block, the design of a re-usable analog IP and finally the organization of a design flow centered on an IP library. After a conceptual overview, this paper presents the methodological principles and details examples with a tutorial intention. The objective is to guide the designer involved in the process of developing analog IPs and corresponding design flow. The method is inspired from platform-based design and adapted here on an original case study: the design of full-custom neuromimetic integrated circuits, built from specific analog computational blocks. The development of re-usable IPs represents an additional effort, mainly for behavioral modeling and characterization. Nevertheless, the steps illustrated in this case study show that the extra time provides a definite advantage for future design projects.
\end{abstract}

Index Terms-Analog_circuits, CAD, Design Re-use, Neuromimetic Circuits and Systems, Behavioral and multidomain simulation.

\section{RE-USE METHODOLOGY FOR CIRCUIT AND SYSTEM DESIGN}

$\mathrm{T}$ HANKS to the growing improvements in semi-conductor manufacturing processes nowadays, VLSI circuits can reach a high level of complexity. The design methodologies have also considerably evolved to allow System-on-Chip (SoC) integration. The SoC design flow is mainly based on reusable components, called Intellectual Property (IP) blocks or cores, which are now well defined and widely used in the digital domain [1]. They are classified from Soft to Hard IP; Soft IP refers to synthesizable HDL (Hardware Description Language) description and a Hard IP is a layout level design. The Firm IP corresponds to an intermediate level, and refers to a pre-synthesized block, using generic components, with a remaining degree of optimization respecting the technology specifications [2]. The advantages of Soft IPs are flexibility and portability, but their real behavior depends on the physical implementation; at the opposite, Hard IPs are optimized for a specific application, on a target technology, consequently they have a predictable behavior.

Moreover, digital design flow is much more automated than the analog one. Digital design profits from standardized tools and methods which allow automatic synthesis. While the trend over the past years has been to integrate both digital and analog circuits on the same chip, the analog part is always the most costly in terms of design effort.

Fig. 1 was extracted from a report on Design topic of International Technology Roadmap for Semiconductors (ITRS) [3]. It illustrates the traditional V-cycle of system design, from specification to layout, with intermediate abstraction levels called behavioral and circuit levels. The topdown way is known as synthesis flow and the bottom-up way as verification flow. This compact picture summarizes the maturity difference between digital and analog design flows and emphasizes future requirements for Electronic Design Automation (EDA). For digital circuits there is a lack of methods and tools at the highest level of the hierarchy, (executable specifications and whole system verification procedures for example); for analog circuits, complete solutions are rarely found at all level of abstractions. Automation of the analog design process is still an open challenge, and re-use methodology is a predominant recommendation.

Important conceptual advances have been proposed in the last twenty years, with precursor works of [12] and [15], resulting in effective software solutions. Our work was guided by these concepts and developments, and also took into account the specificities of neuromimetics circuits and systems. Our background material results from a 20 years experience in our research group in this field of neuromorphic engineering [4] [5]. It consists in particular in a library of analog computation blocks used to design analog ASICs (Application Specific Integrated Circuit) that mimic the dynamics of biological neurons electrical activity [6]. Those blocks were initially described by their electrical schematic and physical layout; we name it the "initial Hard IP." Starting from this background material, we intended to build a dedicated design platform, to automate the design of future neuromimetic ASICs. As demonstrated later, this context is very favorable to the development of a library of Firm IPs, and finally the implementation of an IP-based design flow. 
The paper is organized as follows: in section II, a state-ofthe-art review describes the main concepts and solutions regarding analog design synthesis, and points out issues related to IP re-use in the analog domain. Preliminary tasks for the implementation of the design flow are discussed in section III. Then, we define our methodology in 3 points: the IP-package content (section IV), the design of a re-usable IP block (section V) and the integration of an IP in the system design flow (section VI). Concrete application of the methodology is given in section VII, through examples of design projects [4].

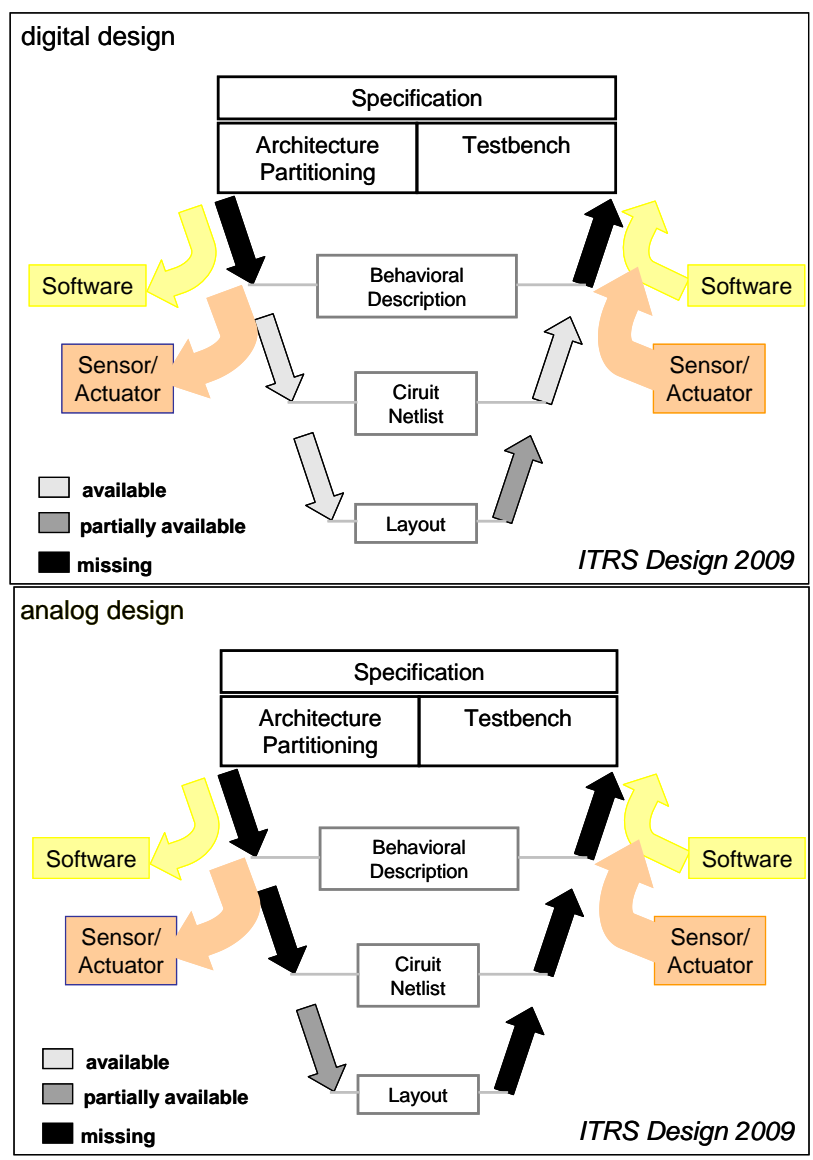

Figure 1. System-Level Design Potential Solutions for digital and analog flow from ITRS Design 2009 [3]

\section{STATE-OF-THE-ART REVIEW : BACKGROUND CONCEPTS AND ISSUES FOR ANALOG IP RE-USE}

\section{A. Background concepts for analog design flow automation}

Before addressing the specifications of an analog IP-based design framework, let us overview the possible approaches to improve the analog design flow for VLSI and reach the automatic analog synthesis.

First, directly inspired from the success story of digital design using FPGAs, we would like to mention the FPAA (Field Programmable Analog Array) technology [7]. Its principle is very efficient for the rapid prototyping of state-of- the-art analog functions; it can't be yet applied to our application domain, which requires very specific functional blocks.

Then, concerning analog ASIC design automation, the main problem is the optimization of one architecture or topology for a set of specifications. Two ways are then possible for this optimization: simulation-based [8], [9] or knowledge-based. The first one sizes a given schematic by a closed-loop process with an optimizer, an analog simulator and standard transistor models. The drawbacks are intensive simulations, CPU time and the selection of the design parameters among a huge list. Analog designers are generally more interested in knowledgebased optimization, to keep control over the choice of parameters. This was also our choice. A review of knowledgebased synthesis tools can be found in [10]. In such an approach, the designer must identify (i) a useful subset of design parameters in relation to the targeted performances, (ii) the circuit equations, and (iii) the sizing procedure.

The circuit equations are supposed to relate the performance parameters to the design parameters. This point is a non-trivial issue, which involves simplification algorithms and more or less insights in the circuit operation [11], [12], [13]. As explained in section IV, our approach leads to multi-level modeling, starting from the ideal functional equation (given by neuroscientists in our case), with gradual refinement to behavioral equations. We use mainly the technique of userdefined equations and sometimes a simulation-based technique coupled with regression.

The circuit sizing process generally happens after topology selection. It starts from an un-sized schematic and follows the designer's steps to set a sized schematic, responding to the specifications. Layout generation, as the final design step, is often a separate task and rarely included in the sizing procedure [14].

For complex integrated circuit, up to the system level, a hierarchy of abstraction levels must be defined and the topdown/bottom-up design flows may be combined in different ways. One can propagate the constraints down starting from top-level to the schematic and layout [15], [16], [17]. Another solution is to merge top-down and bottom-up flows in a meetin-the-middle approach, which is the principle of platformbased design [18], [19]. This method relies on a library of components, well characterized and abstracted in a set of models, used for a bottom-up phase. Then the top-down phase consists in selecting the optimal component, according to some cost function, that satisfies all constraints.

Two main reasons justified our choice to build such a design platform. The first one is related to our specific application field of neuromimetic ASICs. Such devices require the use of non-traditional analog functional blocks, different from OTAs or OPAMPs. In addition, to ensure stability throughout longterm projects in this field, we could start from functional modules already identified and sized for previous projects [6]. 
So we did not question the sizing procedure from the beginning but rather decided to characterize the ability to reuse these blocks; in other words, the question was how to transform a library of Hard IPs (schematic and layout) in a library of Firm IPs and how to implement an automated flow for further design projects.

\section{B. What kind of IP for an analog block?}

The notion of analog IP for automation of analog design flow was the subject of numerous debates [20], [21], [22], [23] and [24].

Ideally, the analog design flow should be identical to the digital one, but this is not the case yet. Of course, the initial context is different and the rules based on analog design are more difficult than Boole algebra. The designer has to challenge non-linear functional relationships, technological parameters and various second order sensitivities [25], [26] (temperature, noise, mismatch, etc.). Analog signal processing is more performing in terms of speed and power consumption but its design is more difficult, closely linked to designer's knowledge. An analog block is designed for a specific application. Consequently there is no consensus on the analog design processes and the primitive functions could be very different depending on cases: current or voltage design mode, bipolar or CMOS design, strong or weak inversion mode for CMOS transistors, etc.

A clear definition of what should contain an analog IP is therefore the starting point for a productive, capable reusebased design framework for analog ICs. The 3 classes of digital IPs have been exposed in the introduction. Let us see hereafter, the pertinence of this classification for the analog case.

\section{1) Hard IP?}

Among the typical operators in analog design, we can find operational amplifiers, ADC and DAC convertors, PLLs, filters, voltage references and RF modules. These blocks are usually optimized in consumption and area for one specific application: the natural trend in analog design is then Hard IP. This type of IP had been chosen in the guide of design and documentation steps by VSIA consortium in 2001 [27].

In this case, technology migrations are solved by re-design instead of re-use.

\section{2) Soft IP?}

What could be a Soft-IP in analog design? We can describe in HDL design language one analog block with the selected functionality. The issue is that there is currently no universal synthesis process which delivers a sized electrical schematic for one application. Furthermore, we first have to select a design mode (current mode, voltage mode), choose primitive functional blocks and select one topology for each. One helping tool capable of enabling the designer to choose one topology among different possibilities would be a great step for the analog synthesis. Promising work about analog circuit sizing method using interval analysis could solve this issue
[28] and allow selecting or deleting some topologies. Nevertheless, before creating this selection tool, a database should be build, with adequate documentation for each topology.

\section{3) Firm IP?}

Between the Soft and the Hard-IP, we find the Firm-IP which seems to be the best choice for analog design. It is a compromise between a fixed layout and a purely HDL description. It proposes a design based on electrical schematic, with enough tunable characteristics to be re-usable for several applications.

\section{State-of-the-art review}

Questions arising from the development of an IP-based analog design flow can be illustrated by Fig. 2. The IP Library is the central point; it is filled by the Analog IP Design Flow and it interacts with the System Design Flow. Then, the first task is to define (1) what kind of information should be embedded in the IP and in which format. After that, it is important to emphasize (2) the design methodology of a reusable IP, because it differs from the design methodology of a "one-shot" circuit. And the last point is (3) the re-use of an IP in the system design flow.

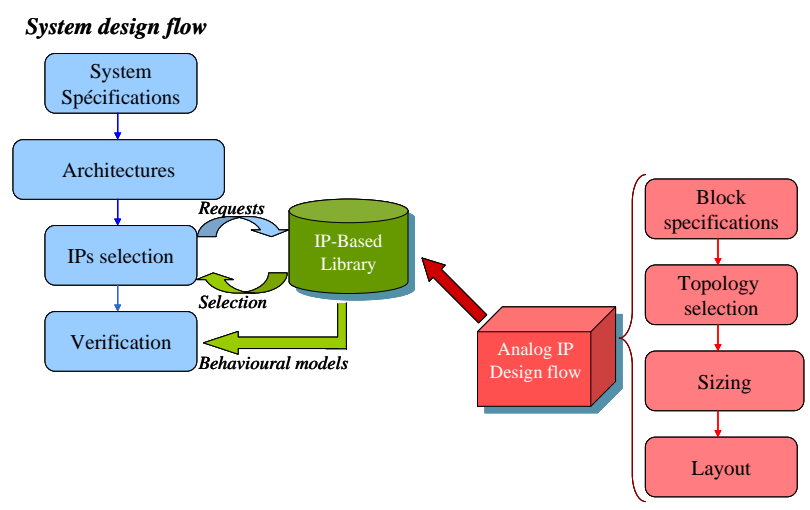

Figure 2. First-preview of an analog IP-based design flow

First, we propose to review how these three points have been addressed by the main contributions that could be found in the literature.

\section{1) IP-package content}

This point deals with the IP general information, including characteristic parameters, design knowledge, models, description formats adapted to every step of the design flow (architecture simulation, sizing, verification, and layout).

O'Connor et al. [29], [30], [31] have defined as analog IP content:

- Set of descriptive views: input/output ports, functional model, structural model

- Set of parameters: for electrical performances, electrical and physical design

- Set of relationships and calculation methods between these parameters.

Evaluation methodology allows the translation from 
physical to performance parameters. Extraction methodology allows the translation from simulations to performance parameters. Synthesis methodology allows the translation from performance to design parameters. Constraint distribution methodology formalizes the translation from IP to under-block specifications.

These relationships are built from the designer knowledge and the main challenge is to express it. This knowledge could be written in an analytical form with an approach based on experiences, or in a procedural form with an approach based on extensive simulations and optimization. This analog IP definition has been applied to amplifier circuits included in a chain of CMOS photo-reception and used to demonstrate the possibility of an automated hierarchical synthesis, in the field of interconnection optical systems.

Secondly, Castro-Lopez et al. [17], [32] have defined an analog IP that contains a behavioral model, a sized schematic and a layout. As this IP is developed for a specific technology, this definition is close to what we consider as Hard IP.

Third, Hamour et al. [33] have proposed an IP definition including similar views: sized schematic, behavioral model, test bench model, but no layout.

Pimentel et al. [34] describe the content of analog and mixed signal IPs: one IP could be divided into three parts: models (functional, behavioral and structural), documents (basic information, user, creation and test guides, physical implementation and models) and circuit schematics.

2) The design of one IP

This point is important for guiding the designer in the different development steps of a re-usable block [35]. Analyzing the experience design to make it re-usable is a complex task. In particular, formalizing the sizing process is a critical step.

Louërat et al. have developed a tool named CAIRO+, which is a platform of analog IP re-use [36], [37]. First, the designer has to describe his circuit into an electrical schematic, a set of sizing parameters and a floor plan layout. CAIRO+ tool can make an automatic sizing and layout, depending on technological parameters, if the procedural sizing steps have been described beforehand. This task is assisted by a synthesis methodology developed in the dissertation of R. Iskander [10].

3) Integration of an IP in the system design flow

What are the main issues related to this point? First, from the architecture exploration to the verification step, it is mandatory to manage different abstraction levels of the IP. Another point is the storage of IPs into a data base which could be queried according to an adequate exploration method.

Besides these questions, interesting approaches of Firm IP hardening flow have been developed, by two previously cited teams. With Louërat et al., the IP description in CAIRO+ is parametric and described into equations then the Hard IP is automated and needn't optimization. Some examples are in the Nguyen-Tuong dissertation [14]. With O'Connor et al., the synthesis process is described with UML language and it is included into the design platform (RUNE II) which contains simulation and optimization tools [38].

Note that, despite the general trend is to design a library of Firm IPs, a specific adjustable functional component, the answers to the previous three main questions depend on the initial IP point of view. The method used to reach the desired Firm IP differs in two ways: in some cases the notion of Firm IP is progressively derived from a Soft IP view, while in other cases it is derived from an initial Hard-IPs library. Our approach belongs to this second case. Indeed our background material was a library of blocks listing 3 views: symbol, schematic and layout. As explained in section I, the issue here was to transform a library of Hard IPs in a library of Firm IPs. The critical points in the proposed process are: multi-level modeling, characterization of validity range to fit requested performances. Finally we implemented a system design flow, based on the automated exploration of an IP database. We are now going to detail step by step the process methodology.

\section{PRELIMINARY TASKS TO ESTABLISH THE DESIGN FLOW}

Preparatory tasks are required to structure and finally establish an IP-based design flow. The first one is to define the system hierarchy and the primitive blocks, which are associated to each level of this hierarchy. The second one is to define a set of typical specifications that constrains the circuit performances. This part will describe these preliminary issues, with examples taken from our application context of neuromorphic engineering.

\section{A. The specific context of neuromorphic engineering}

The originality of that work relies on this particular application domain: the design of analog ASICs that are involved in neuromorphic systems. The goal of neuromorphic engineering is to develop integrated circuits and systems that emulate the electrical activity of biological neural networks. From the microelectronic point of view, one solution is to design analog ASICs for real-time computation of neurons' activity and to digitally control the connectivity between these neurons. We focus here on a series of analog ASICs that implement an electro-physiological neuron model, inspired from the Hodgkin-Huxley $(\mathrm{HH})$ formalism [39]. They are typically used as hardware simulators in computational neurosciences, in order to explore information coding and adaptation principles in the brain [40].

This domain has appeared to be a favorable background to the development of a design flow based on IP-reuse. The main reason is that the bases of system hierarchy and design primitives have been clearly established, along successive design projects [5]. This point is finally similar to the reality of digital design and it should be the first requirement of the methodology: what is the system hierarchy and what are the 
primitives at each hierarchical level?

\section{B. Design primitives and system hierarchy}

The principle of the modular design of the neuromimetic ASICs has been already exposed in [6]. The architecture of one ASIC is based on the repetition of a small number of computation primitives, namely sigmoid, kinetic, power, output (see Fig.3). One can consider them as a first version of the IP blocks, on the form of Hard IPs, that were realized on AMS BiCMOS $0.35 \mu \mathrm{m}$ process. Each of these elementary cells is an analog operator involved in the equations of the Hodgkin-Huxley formalism. These analog operators are then assembled to produce a so-called neuromimetic ASIC, capable of emulating, in real-time, the dynamics of a small neuron's network.

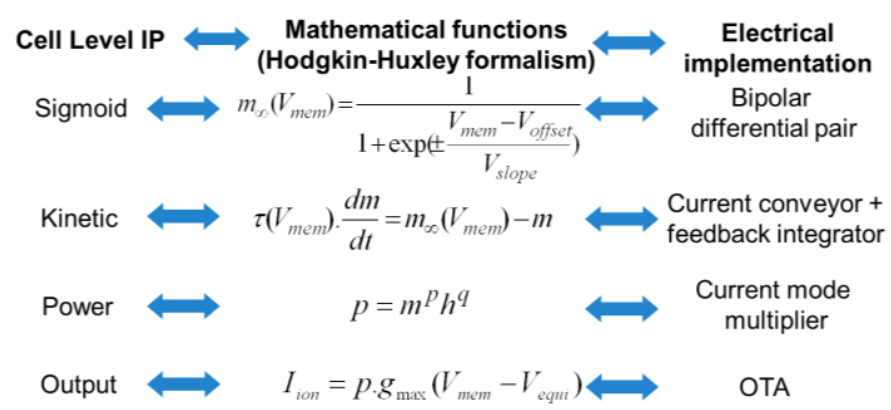

Figure 3. Correspondence between mathematical function and electrical implementation, for each type of Cell Level IP. All the voltages $\left(V_{m e m}, V_{\text {offset }}\right.$, $\left.V_{\text {equi }}, V_{\text {slope }}\right)$ are in Volt in biological expression and are multiplied by 5 for electronic correspondence. The time constant $(\tau)$ is the same in electronical and biological fields. The conductance $\left(g_{\max }\right)$ is in Siemens in biological expression and multiplied by 10 in its electronic counterpart. $m$ is a probability (unitless) in biological model and it is a current (Ampere) in electronic. $p$ and $q$ are integers in both electronic and biological expressions.

Fig. 3 shows the correspondence between the mathematical function of the $\mathrm{HH}$ model and the analog cells that are used for its realization. Note the choice of a current mode design. $V_{\text {mem }}$ is the membrane potential of the neuron, while $V_{\text {offset }}$ and $V_{\text {slope }}$ are input voltages used to tune the neuron model parameters. $\tau$ is the kinetic of the activation/inactivation of ionic current. $g_{\text {max }}$ is the maximum conductance, and $m$ and $h$ represent the activation and inactivation terms, respectively. Dynamic functions describe the permeability of membrane channels to its specific ion. $V_{\text {equi }}$ is the ion-specific reverse potential and $p$ and $q$ are integers. $m$ relaxes back toward its associated steadystate value $m_{\infty}$, which is a sigmoid function of $V_{\text {mem }}$.

Starting from the previous basic operators, it is possible to construct the hierarchy of an integrated circuit that emulates a biologically realistic neural network: this hierarchy is described on Fig.4. The IP level corresponds to the recurrent computation blocks of Fig. 3 and the higher hierarchical levels of rank $\mathrm{N}+1$ are simply structural descriptions using the primitive blocks of level $\mathrm{N}$.

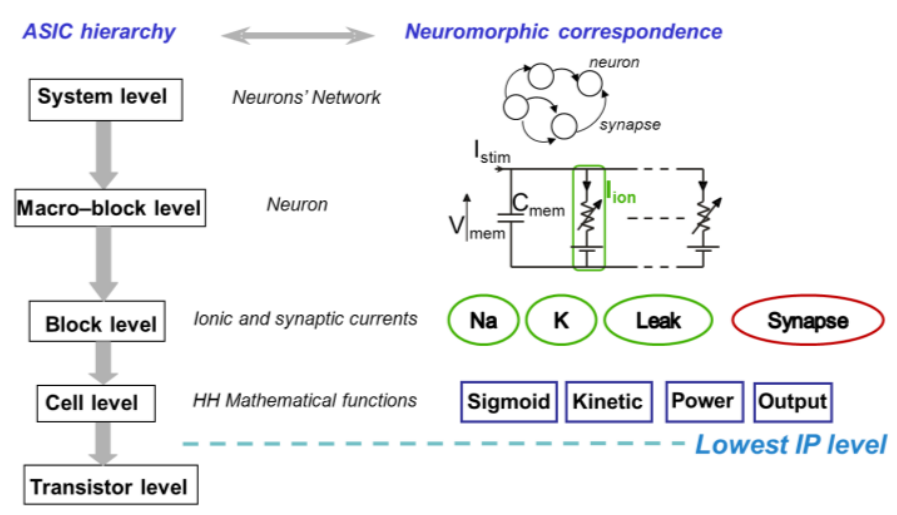

Figure 4. ASIC hierarchy and related neuronal computation blocks

\section{System specifications and IP-block performances}

The specifications of such a system are slightly non-typical, regarding the traditional analog design field. The neuroscientists interested in those systems want to fix:

- the number of neurons defining the network: $N$ (defined at System Level)

- the number and type of ionic channels considered for each neuron: $\mathrm{Na}$ conductance (Sodium channel), $\mathrm{K}$ conductance (Potassium channel), Leak conductance (Leak channel) and Slow conductance (additive channel for calcium dependence)

(defined at Macro-block level)

- the number of synapses $N s$ for each neuron and their electrophysiological parameters: $\tau, V_{\text {equi }}$ and $g_{\max }$. (defined at Macro-block level)

- the electrophysiological parameters of each ionic channel: $V_{\text {offset }}, V_{\text {slope }}, \tau, V_{\text {equi }}$ and $g_{\text {max }}$. (defined at Block Level and propagated to Cell Level). Let us note that for the $\mathrm{Na}$ conductance and Slow conductance, the parameters $V_{\text {offset }}, V_{\text {slope }}$ and $\tau$, are duplicated because of two concurrent processes in the neurophysiology, named activation and inactivation.

A typical specifications set is given on Table I. It corresponds to a small circuit of two neurons: Neuron 1 has 3 ionic channels $(\mathrm{Na}, \mathrm{K}$, Leak) and Neuron 2 has one additive channel for calcium dependence (Slow conductance). 
TABLE I

TYPICAL SPECIFICATIONS OF AN ASIC WITH A SMALL NETWORK OF 2 NEURONS

\begin{tabular}{|c|c|c|c|c|c|c|c|c|}
\hline & Voffset_act & Vslope_act & Voffset_inact & Vslope_inact & $\tau \_a c t$ & $\tau$ _inact & Vequi & $g \max$ \\
\hline Sodium & $-39 \mathrm{mV}$ & $8,55 \mathrm{mV}$ & $-47 \mathrm{mV}$ & $-7.3 \mathrm{mV}$ & $0.03 \mathrm{~ms}$ & $0.25 \mathrm{~ms}$ & $40 \mathrm{mV}$ & $0.7 \mu \mathrm{S}$ \\
\hline Potassium & $-27 \mathrm{mV}$ & $12,6 \mathrm{mV}$ & & & $3 \mathrm{~ms}$ & & $-80 \mathrm{mV}$ & $1.4 \mu \mathrm{S}$ \\
\hline Leak & & & & & & & $50 \mathrm{mV}$ & $0.5 \mu \mathrm{S}$ \\
\hline Synapse & & & & & $4 \mathrm{~ms}$ & & $60 \mathrm{mV}$ & $0.3 \mu \mathrm{S}$ \\
\hline Synapse & & & & & $5 \mathrm{~ms}$ & & $50 \mathrm{mV}$ & $0.2 \mu \mathrm{S}$ \\
\hline
\end{tabular}

\begin{tabular}{|l|l|l|l|l|l|l|l|l|}
\hline & Voffset_act & Vslope_act & Voffset_inact & Vslope_inact & $\tau_{-}$act & $\tau$ _inact & Vequi & gmax \\
\hline Sodium & $-39 \mathrm{mV}$ & $8.55 \mathrm{mV}$ & $-47 \mathrm{mV}$ & $-6,6 \mathrm{mV}$ & $0.03 \mathrm{~ms}$ & $0.25 \mathrm{~ms}$ & $40 \mathrm{mV}$ & $0.7 \mu \mathrm{S}$ \\
\hline Potassium & $-27 \mathrm{mV}$ & $12.6 \mathrm{mV}$ & & & $3 \mathrm{~ms}$ & & $-80 \mathrm{mV}$ & $1.4 \mu \mathrm{S}$ \\
\hline Slow conductance & $-55 \mathrm{mV}$ & $10.4 \mathrm{mV}$ & $-38 \mathrm{mV}$ & $-20 \mathrm{mV}$ & $6 \mathrm{~ms}$ & $6 \mathrm{~ms}$ & $-100 \mathrm{mV}$ & $0.1 \mu \mathrm{S}$ \\
\hline Leak & & & & & & & & \\
\hline Synapse & & & & & $60.33 \mu \mathrm{mV}$ & & $0.1 \mu \mathrm{S}$ \\
\hline
\end{tabular}

Furthermore, the implemented neuron models must emulate a variety of biological neuron behaviors, thus the IP-blocks must be designed to guaranty a certain tuning range for the electrophysiological parameters. This constraint imposes that the IP-block functionality must be preserved while varying the electrophysiological parameters in the so-called validity ranges. Hence, the performances of the IP-blocks are quite non typical and are given in terms of these validity ranges. For example, typical performances for sigmoid IP are given in Table II, for kinetic IP the time constant range have to be $[\tau, 50 \tau]$ and for output IP the transconductance range $\left[g_{\max }, 50 g_{\max }\right]$.

TABLE II

TYPICAL PERFORMANCES OF SIGMOÏD IP, IN THE FORM OF VALIDITY RANGE FOR INPUT VOLTAGES WITH A SUPPLY VOLTAGE OF $5 \mathrm{~V}$

\begin{tabular}{|l|l|l|}
\hline $\begin{array}{l}\text { Performances } \\
\text { parameters }\end{array}$ & Minimum & Maximum \\
\hline$V_{\text {mem }}$ & $0.5 \mathrm{~V}$ & $4.5 \mathrm{~V}$ \\
\hline$V_{\text {offset }}$ & $1.5 \mathrm{~V}$ & $3.3 \mathrm{~V}$ \\
\hline$K_{\text {slope }}$ & $1.7 \mathrm{~V}$ & $4.7 \mathrm{~V}$ \\
\hline
\end{tabular}

After this introduction, required to efficiently prepare the implementation of an IP-based design flow, we will now consider in details the three main points of the methodology.

\section{ANALOG IP PACKAGE}

As explained in part II, our approach is to build Firm IPs starting from Hard IPs (only symbol, schematic and layout) that have previously been designed and optimized for a given project. So the question is: how can we transform a Hard IP to a Firm IP? Based on our experience, combined with the proposed studies in the literature, it appears that the main properties required for a re-usable IP are:

- ease of simulation at each level of abstraction of the system,

- description of the design process and

- ability to resize the IP to deal with a technology migration or a change in specifications.

In our methodology, each Firm IP we created has 7 informative views that are described in Table III. The views Symbol, Schematic and Layout were already present in the Hard IPs library. The new Firm IP package contains additive description views that correspond to hierarchical abstraction models (Connectical, Functional and Behavioral). These models are developed by the designer, in a standard design language. In our case, we use the Verilog-A language [41]. In order to have a fluent design flow, all these views have the same terminals and the same symbol as the schematic. These views are useful for multilevel simulations especially in the verification phase of the design process. The Functional view describes the ideal equations of the function to be implemented. The Behavioral view is more detailed and contains refined equations that fit the circuit non-ideal behavior [42], [43]. The Connectical view is an empty model used to verify that blocks are well connected, without any functional equations. It is used for the ultimate verifications, when the overall ASIC needs to be simulated.

TABLE III

INFORMATIVE VIEWS EMBEDDED IN THE ANALOGUE IP PACKAGE

\begin{tabular}{|c|c|c|}
\hline View name & Description and role & Format \\
\hline Symbol & visualizes the function & graphical \\
\hline Connectical & $\begin{array}{l}\text { verifies connection between } \\
\text { blocks }\end{array}$ & Verilog-A \\
\hline Functional & models ideal electrical behavior & Verilog-A \\
\hline Behavioral & 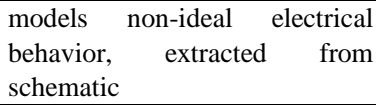 & Verilog-A \\
\hline Schematic & transistor-level schematic & $\begin{array}{l}\text { graphical / } \\
\text { netlist }\end{array}$ \\
\hline Layout & $\begin{array}{l}\text { physical design in targeted } \\
\text { technology }\end{array}$ & $\begin{array}{l}\text { graphical / } \\
\text { GDS-II }\end{array}$ \\
\hline Documentation & design knowledge formulation & text \\
\hline
\end{tabular}

To emphasize the re-usability of one IP, we also add a last file, namely the Documentation view. This file finally collects the design expertise and contains data such as:

- the performance and design parameters

- the design procedure and sizing algorithm (or how to link the performance parameters to the design parameters)

- the characterization of the block with the objective of reuse (what are the performances, how to measure them, 
and how far the circuit functionality is stable regarding operational changes)

- information that concerns the already designed layout view (technology, supply voltage, area and cost).

\section{DESIGN OF A RE-USABLE IP}

Design for re-use is not limited to the design and sizing of a "one-shot" circuit (Hard IP case). An additional effort is required to obtain a Firm-IP; in our case it is to express the design procedure, to develop hierarchical models, to characterize the circuit and to write the documentation file. This methodology is presented here using the example block sigmoid.

\section{A. Design procedure of one IP}

The expression of design expertise is essential to make an IP re-usable. The topology of the circuit being given, we must begin by identifying the performance parameters of the block (the parameters that are constrained by specifications) and the design parameters (those that can be adjusted in the design procedure); then explain how the design parameters are linked to the performance parameters. Let develop this point on the sigmoid block.

As mentioned in Fig. 3, the mathematical function of the $\mathrm{HH}$ formalism to imitate is the following:

$$
m_{\infty}\left(V_{\text {mem }}\right)=\frac{1}{1+e^{\frac{-(\text { Vmem-Voffset })}{\text { Vslope }}}}
$$

In practice, one chooses to represent the variable $m_{\infty}$ with a normalized current $2 I_{0}$. The electrical functional equation becomes:

$$
I_{m_{\infty}}=\frac{2 I_{0}}{1+e^{\frac{-(\text { Vmem-Voffset })}{\text { Vslope }}}}
$$

The topology chosen to emulate this function is based on a differential pair which takes direct input voltages $V_{\text {mem }}$ and $V_{\text {offset }}$. The slope control parameter $V_{\text {slope }}$ of the model is generated through a current-voltage converter, controlled by the $K_{\text {slope }}$ voltage [5]. The Schematic view is given below by Fig. 5:
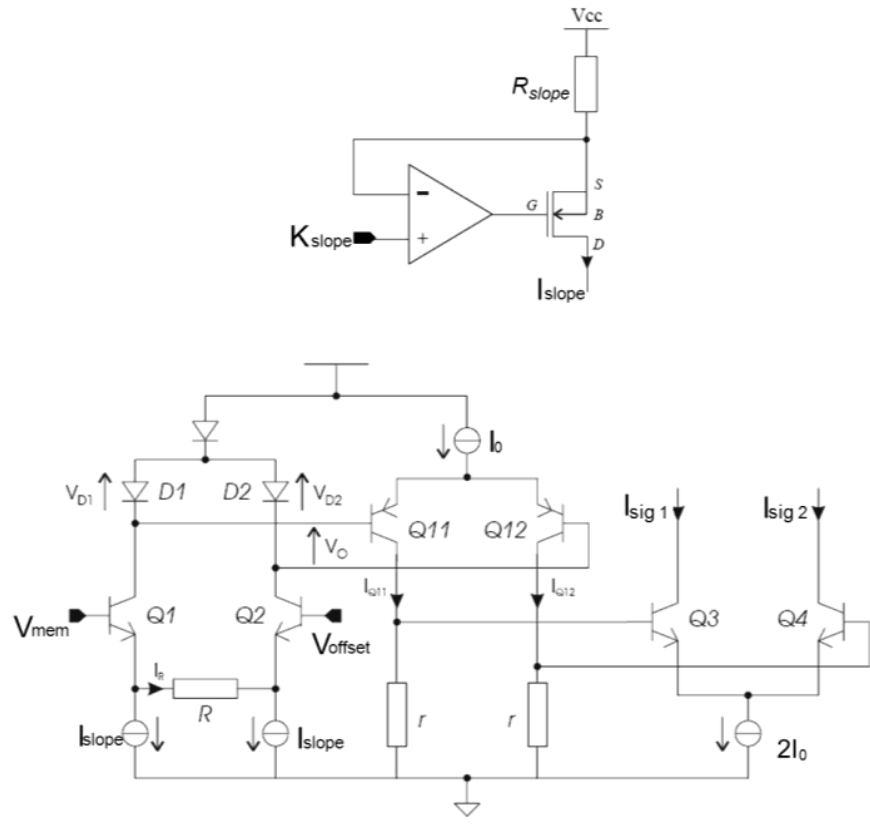

Figure 5. Schematic view of sigmoid Cell level IP

The first and second sub-blocks are finally described by the following functional equations:

$I_{\text {slope }}=\frac{V c c-K_{\text {slope }}}{R_{\text {slope }}}$

$I_{\text {sig } 1,2}=\frac{2 I_{0}}{1+e^{\frac{r \cdot I_{0} \cdot(\text { Vmem-Voffset })}{R \cdot U_{T} \cdot I_{\text {slope }}}}}$

Thus the tuning of the $V_{\text {slope }}$ parameter is made using the following equation where $U_{T}$ is the thermal voltage $(\approx 25 \mathrm{mV}$ at $\left.25^{\circ} \mathrm{C}\right)$ :

$V_{\text {slope }}=\frac{R U_{T}}{r I_{0}} \frac{V c c-K_{\text {slope }}}{R_{\text {slope }}}$

We can extract the parameters needed for this design procedure. The performance parameters are $V_{\text {mem }}, V_{\text {offset }}, V_{\text {slope }}$ (or $K_{\text {slope }}$ which is linearly related to $V_{\text {slope }}$ ). The design parameters are: $R_{\text {slope }}, R, r$ and $I_{0}$. The previous equations are used to connect those two sets of parameters using a method based on knowledge.

\section{B. Multi-level modeling of one IP}

As explained previously, models are considered at 3 levels of abstraction and are written in Verilog-AMS. Using the example of the sigmoid block, the Functional model reproduces the functional equations of the preceding paragraph (3), (4), (5). For the Behavioral model, the input and output impedances are included as well as non-ideal effects. For instance, in this sigmoid block, we add the non-ideal gain of the op-amp in the voltage-current converter, the unbalanced differential pairs and the copy factor errors of current mirrors. All that non-ideals characteristics are extracted from electrical simulations of Schematic view. 
The Connectical view is at a higher abstraction level; it is a virtually empty block, simply being responsible for detecting defaults of connection between blocks. The principle consists in verifying that the voltages and currents applied to the block lie within a plausible range, and if so, the output is set to a nominal value, if not it is set to zero.

Simulations with multiple levels of abstraction are then very interesting to optimize the simulation time: some critical IPs can then be represented by their Behavioral or even Schematic view, while standard IPs may be represented by their Functional or Connectical view.
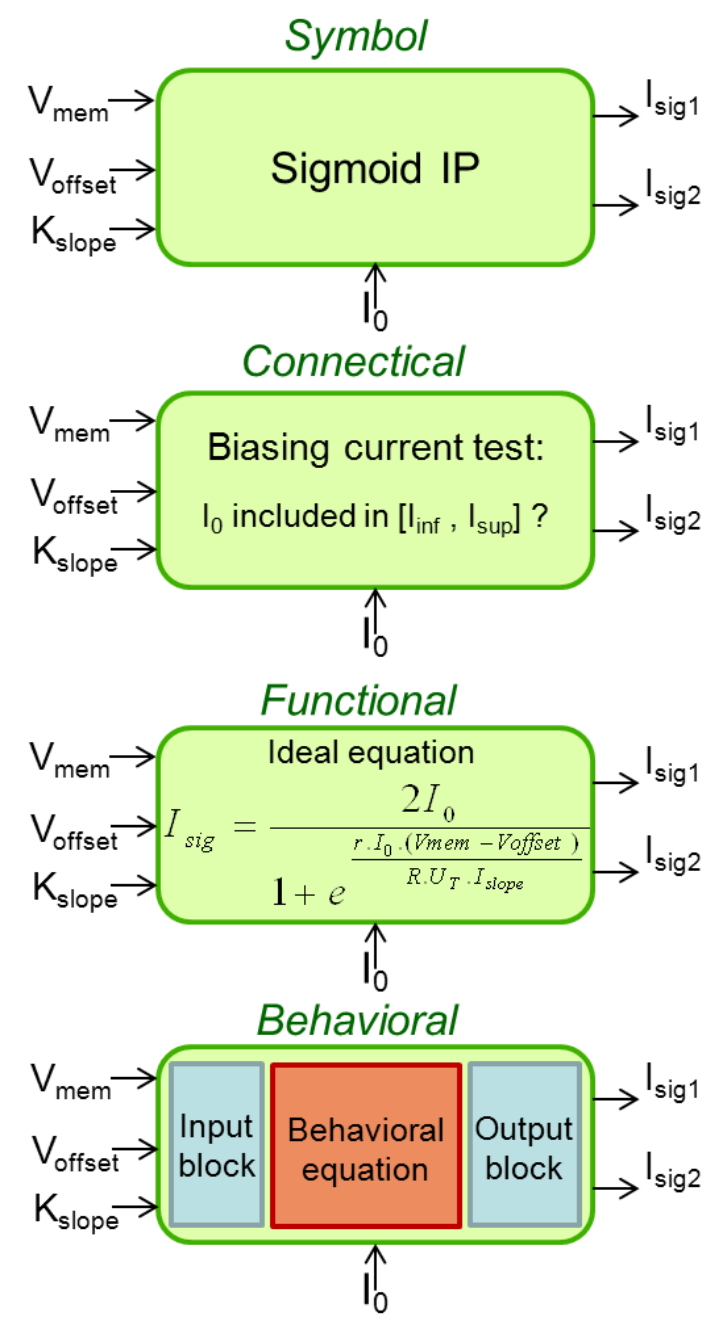

Figure 6. Modelling views

\section{Characterization procedure of an IP}

The IP characterization is useful to define the performances of the cell. In the context of re-use, the question is also: under what conditions the unit may undergo a change in specifications?

We have previously seen that the performance parameters of the sigmoid block are $V_{\text {mem }}, V_{\text {offset }}$ and $K_{\text {slope }}$ input voltages. The IP has been sized once to ensure proper operation for precise values of these voltages. To what extend can the IP keeps its functionality for a different set of values? To determine this, a systematic analysis was performed to extract the validity ranges of the performance parameters, i.e. the intervals of $V_{\text {mem }}, V_{\text {offset }}$ and $K_{\text {slope }}$ where the sigmoidal function is preserved. This study is conducted by performing a series of simulations (AC, DC, transient and Monte Carlo) with the initial Hard IP, already sized for a given set of specifications. The validity ranges of input voltages of sigmoid block have been previously given on Table II.

This simply means that the IP sigmoid can be re-used without modification in its design, provided that the specifications for $V_{\text {mem }}, V_{\text {offset }}$ and $K_{\text {slope }}$ are included in these intervals.

The Documentation file includes the description of the characterization procedure as well as the results of all simulations. To illustrate this, Fig. 7 shows the responses of the circuit $\left(I_{\text {sig }}\right)$ to a DC voltage sweep of $V_{\text {mem }}$ for several values of $V_{\text {offset }}$.

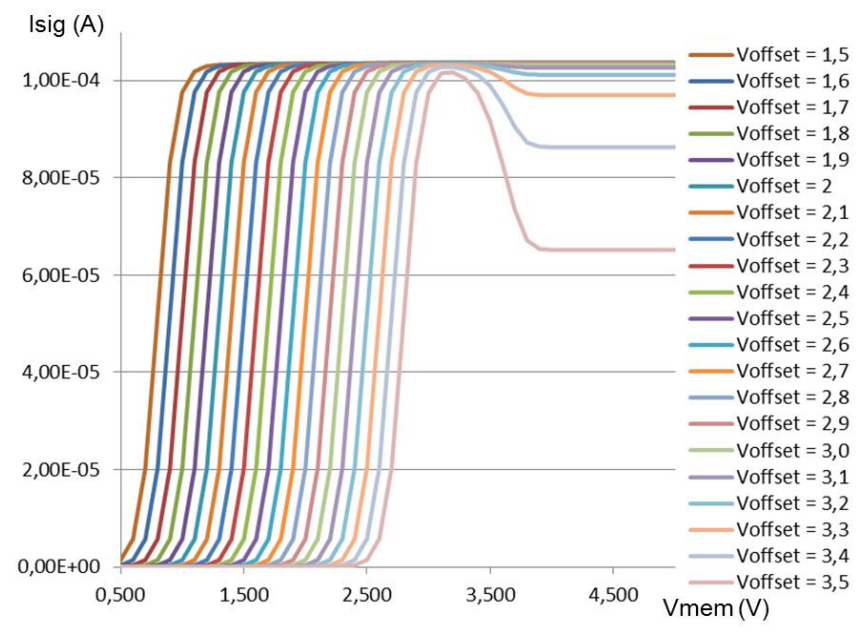

Figure 7. DC current response of Sigmoid cell parametered by $V_{\text {offset }}$

These responses are compared to the ideal response of the sigmoidal function (simulation with Functional view) by measuring the mean square error.

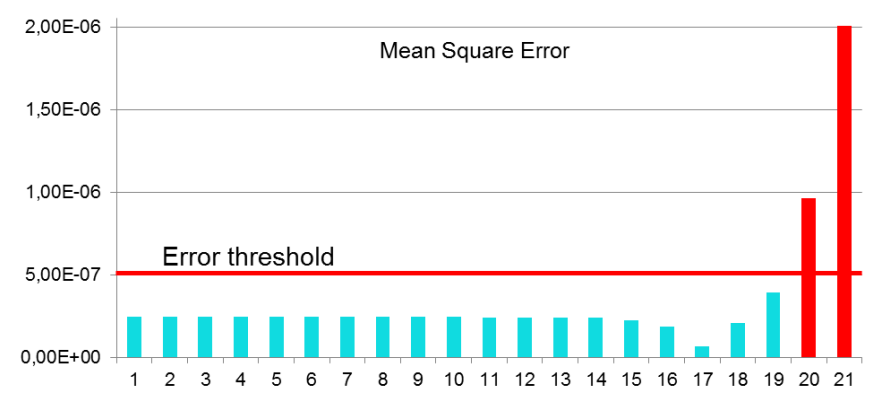

Figure 8. Mean square error of Sigmoid block (schematic view compared to ideal fonctional view) output current, depending on $V_{\text {offset }}$; numbers 1 to 20 correspond to incremented values of $V_{\text {offset }}$ from $1.5 \mathrm{~V}$ to $3.5 \mathrm{~V}$. 
The validity range for $V_{\text {offset }}$ is then determined so that this error does not exceed $5 \times 10^{-7}$. Fig. 8 shows that bars 20 and 21 are outside the range $(20,21$ corresponds respectively to $V_{\text {offset }}=3.4 \mathrm{~V}$ and $V_{\text {offset }}=3.5 \mathrm{~V}$ ); thus the validity range for $V_{\text {offset }}$ is $[1.5,3.3] \mathrm{V}$, as mentioned in Table II.

To complete the IP characterization procedure, we evaluate the robustness of this validity range versus the technological fluctuations. We performed 3 Monte-Carlo simulations using as input values, respectively the middle, the upper bound, the lower bound of the parameters intervals (Table II). Fig.9 gives an illustration of the Monte-Carlo simulation (100 samples) of the response of Sigmoid IP $\left(I_{\text {sig }}\right)$, for $V_{\text {offset }}=1.5 \mathrm{~V}$, lower bound of the preliminary validity range. If the distribution of $I_{\text {sig }}$ values results in more than $5 \%$ out of the interval [0.95*Targeted value, $1.05 *$ Targeted value], we reduce the validity range of the used parameter (in this Fig. 9, $V_{\text {offset }}$ ).

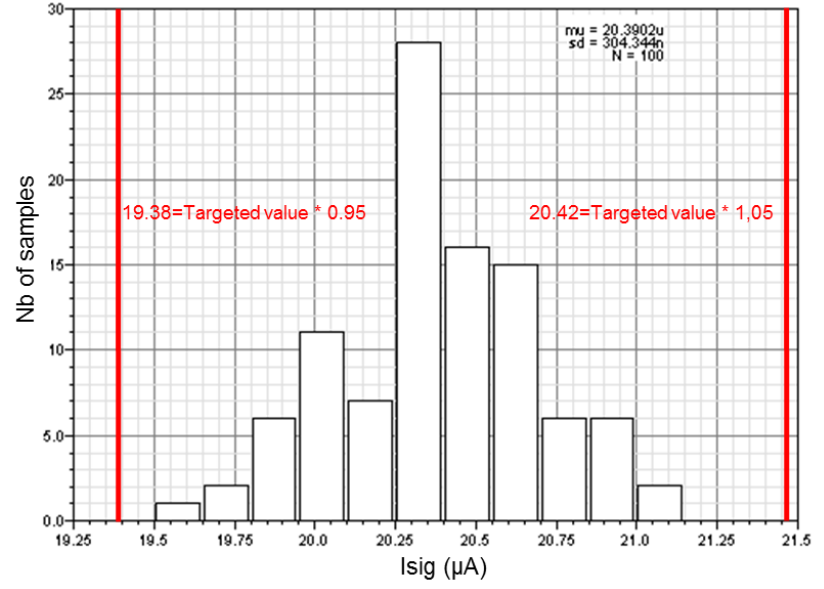

Figure 9. MonteCarlo simulation of the current output Sigmoid IP with parameter $V_{\text {offset }}=1.5 \mathrm{~V}$ (100 samples). Targeted value is $20,40 \mu \mathrm{A}$ (theoritical value), standard deviation is $304,34 \mathrm{nA}$, and all values are inside the tolerance interval $[0,95 * 20,40 \mu \mathrm{A} ; 1,05 * 20,40 \mu \mathrm{A}]$.

In that case, MonteCarlo simulation results show that $0 \%$ of $I_{\text {sig }}$ simulated values are out of the tolerance interval; thus we don't modify the preliminary validity range (defined in Table II).

\section{IMPLEMENTATION OF AN IP-BASED ASIC DESIGN FLOW}

The recurrent IP blocks have been now designed, modeled and characterized using the approach described in the previous paragraph. Now the arising question is the organization of the IPs in a database that can be the hub of the design flow of an ASIC. In this part, the IP database implementation and exploration are addressed.

\section{A. General organization of the flow}

A typical flow chart is given in Fig. 10.

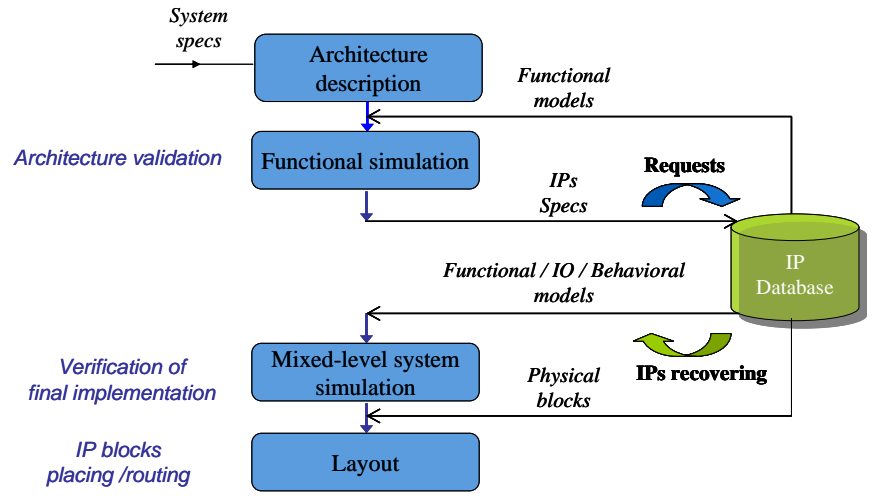

Figure 10. Principle of an IP-based design flow

Once the ASIC specifications are known (see typical specifications of Table I), functional models present in the database can be used to validate a first architecture by highlevel simulations. The database is then queried to see if the IPs can be re-used. If so, the different views contained in the IP package are retrieved for the final stages of the design. If not, we will see how characterization information can be used to re-adjust an existing IP.

\section{B. Database structure}

IPs are gathered in a SQL database hosted on an Apache server [44]. Ten tables were needed to describe this database:

- Definition of each IP: 1 table

- Link father-son between functions (hierarchical link between level $\mathrm{N}$ and level $\mathrm{N}-1$ ): 1 table, describing the hierarchy of the system

- Link father-son between IPs: 3 tables, one for each hierarchical level (Cell, Block, Macro-block)

- Number of IPs for a given function: 1 table

- Performance parameters' values of each IP: 3 tables, one for each hierarchical level (Cell, Block, Macro-block)

- Connection between different Macro-blocks which define the whole system: 1 table

This covers all information for one IP, including characterization and hierarchical data, to be able to implement an automatic top-down exploration. This database is included in an operating platform that transforms the specification (captured on a remote computer by the 'client') in requests (sent to the server machine designer). This required the use of PHP [45], SQL [46], HTML and XML [47].

Once the specifications have been entered in a web form, a PHP script extracts data from it, arrange them in a text file and produce a report for the client. For the designer's point of view, the specification data are transformed into SQL requests which explore the database and a report is created.

\section{Database exploration method}

The most important criteria for the exploration of the database are: (i) the targeted technology and (ii) the desired specifications, in the form of parameter values $P_{\text {spec }}$, such as 
those of Table I: $V_{\text {offset }}, K_{\text {slope }}, \tau, V_{\text {equi }}, g_{\text {max }}$. As explained previously, re-usability of each of the IP is characterized by validity domains $\left[P_{\min }, P_{\max }\right]$ for the performance parameters $V_{\text {offset }}, K_{\text {slope }}, V_{\text {equi }}, g_{\text {max }}$. For the kinetics parameter, $\tau$, it depends on an external capacitance value, connected to the kinetic block.

The exploration method is a Top-Down one, from the Macro-Block Level to the Cell Level; an IP is selected if its performance validity domains can cover the targeted specifications, i.e. if :

$P_{\text {min }}<P_{\text {spec }}<P_{\text {max }}$

The database exploration algorithm is based on the principle illustrated by the chart of Fig. 11. If we have different corresponding IPs, we take the closest to the specifications: we calculate, for each parameter, the distance between the specification and the middle of the validity range, named $d$ :

$d=\left|P_{\text {spec }}-\frac{P_{\max }+P_{\min }}{2}\right|$

Then we accumulate the total distance by adding the contribution of each parameter. It is logical to take the IP corresponding to the minimum total distance.

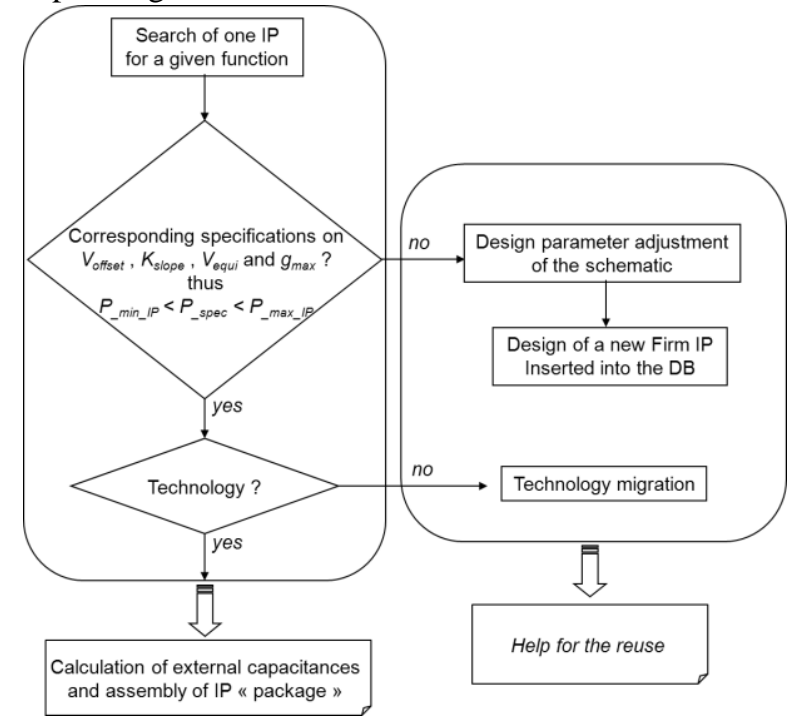

Figure 11. Exploration algorithm used to look for an IP in the database

\section{Results of the exploration}

As illustrated on Fig. 11, when looking for one IP corresponding to given specifications, the answer can be:

- a) one IP exists in the database

- b) no IP could be immediately found but there is one IP whose performances are close to the specifications

- c) one IP corresponds to the specifications but it was designed in another technology process.

In case a), the IP package is recovered by the designer. In cases b) and c), an aid for re-design is given. Case b) will be illustrated in an example in the next part. In the case (c)) the issue results from the technology or the supply voltage, some guidelines we developed for a resizing methodology can help to fix the problem, [48] but that will not be detailed here.

At the final step, a diagnosis is returned and quantifies the possibility of re-using IPs for a new ASIC project. This is illustrated in Fig. 12. In practice, this step is performed thanks to an XML file that summarizes the results of the exploration and allows generating an HTML page (not shown) of the diagnosis. In case of a successful exploration (previous case a)), this page also indicates, the result of the automatic calculation of external capacitances (needed to obtain the desired $\tau$ values) and details the IP content with related links to access more information. 


\section{Exploration Results}

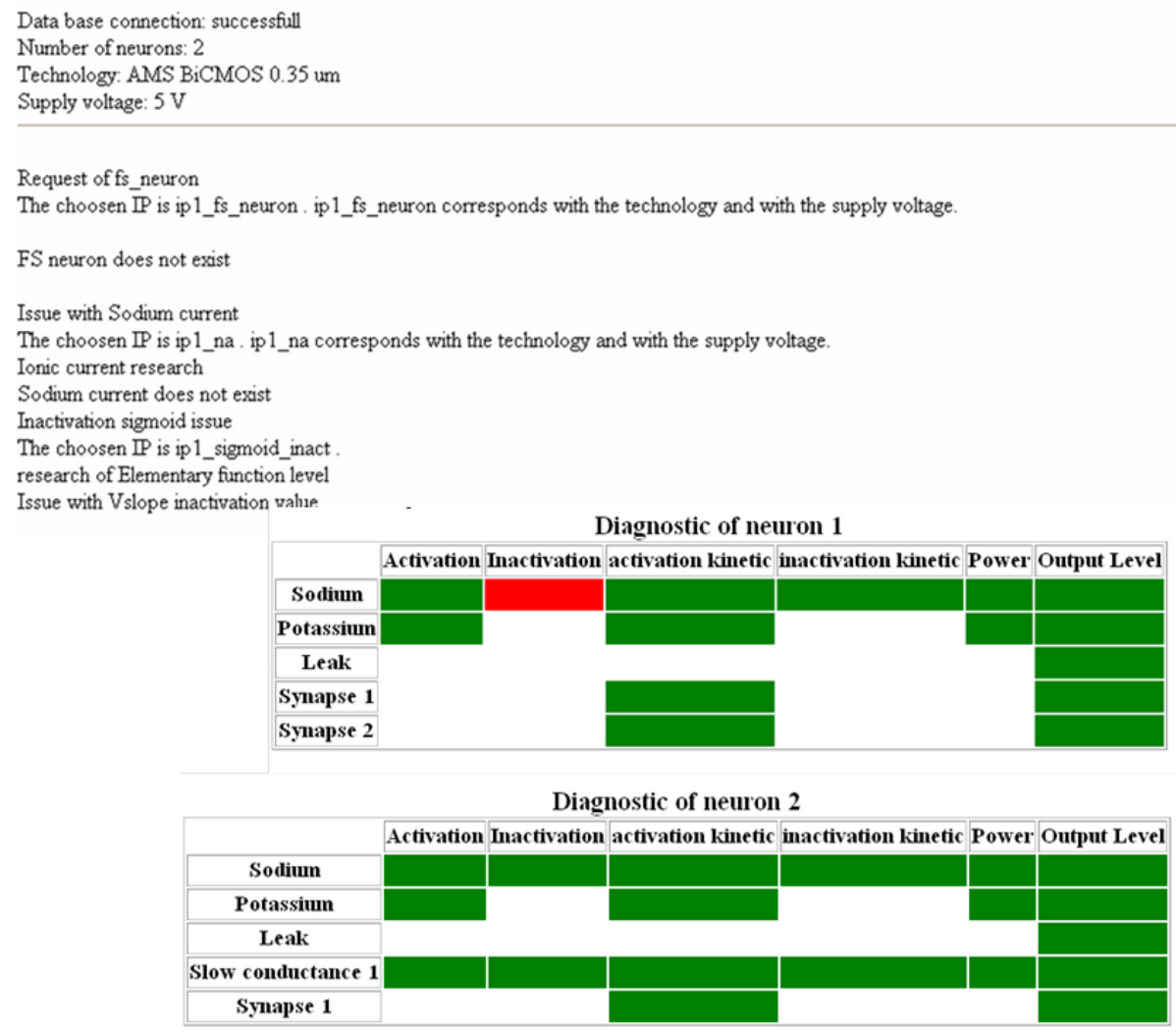

Figure 12. Screen snapshot of HTML diagnosis of re-use for a given ASIC design project ; a green boxt means "the IP has been found with the desired specfications", a red box means "no IP has been found" and a white box reports on a case not applicable. This diagnosis describes firstly the connection status to the database, the number of requested neurons, the chosen technology and associated supply voltage. Secondly, it describes the result of the top-down database exploration. We see in the example that the macro-block FS neuron didn't match all the specifications. Tests to identify the problem source showed that the

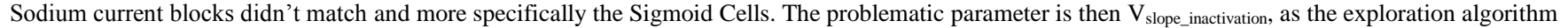
cannot find a corresponding IP. A table summarizes the results: green marks when the IP is identified, red mark otherwise.

\section{EXAMPLES OF USE}

The database automatic exploration process has been tested on many plausible specifications' sets. Here we propose two examples of the implemented design flow, each one corresponding to realistic design projects.

\section{A. Design project 1}

For this first application, the objective is to design one neuromorphic ASIC, which is composed of five neurons: three are regular spiking neurons (RS neuron), one is a fast spiking neuron (FS neuron) and one is a slow conductance neuron (5c neuron) [49]. The initial specifications, defined in agreement with our biologist partners, are partially given on Table I of part III. This Table summarizes the specifications of 2 of the 5 neurons.

As biological specifications are filled in, designer chooses conversion factors (voltage and capacitance factors) as well as the targeted technology to transform biological specifications to electrical specifications.

In this favourable case of use, the diagnosis of re-use indicates a total success, i.e. all needed IPs have been found in the database. The exploration program takes less than one minute to give the result, in the form of 5 diagnosis tables. Then, the designer can quickly retrieve the system netlist, as well as all IP-packages. At this step, the designer is able to perform different verifications through multi-level simulations of the entire ASIC. 


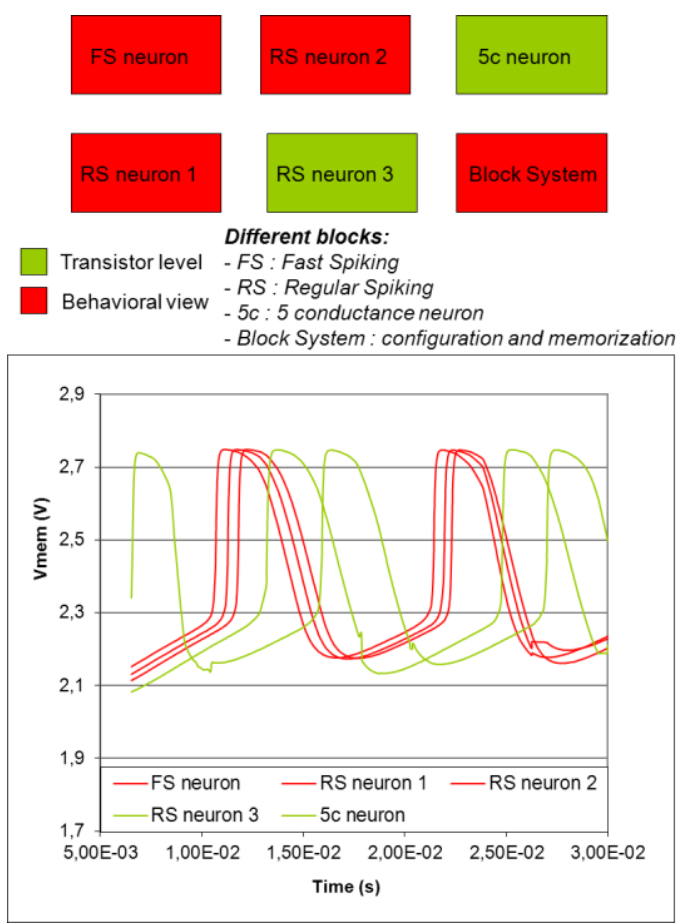

Figure 13. Multi-level abstraction simulation results of one ASIC setting with 5 neurons

To illustrate this, Fig. 13 shows a multi-level simulation of the full ASIC. We test the RS neuron 3 and $5 c$ neuron with transistor level descriptions (Schematic view) while the other blocks use Behavioral views. The validation criterion is the presence of oscillations with neuromimetic spikes on the membrane voltage of each neuron, what appears clearly on the transient response. The Behavioral view realizes a compromise between accuracy and computation time. The simulation of one FS neuron at transistor level takes $14 \mathrm{~min}$ $56 \mathrm{~s}$ for a simulation stop time of $60 \mathrm{~ms}$; this duration decreases to $3 \mathrm{~min} 17 \mathrm{~s}$ using the Behavioral view. Furthermore, this simulation time increases exponentially versus the number of neurons (around 10 hours for a simulation of 5 neurons at transistor level). This design project 1 was complete and was sent to the foundry. This new ASIC named Galway is currently working.

\section{B. Design project 2}

For this second application, the objective is to design one ASIC composed of two neurons. The initial specifications are slightly different from those of Table I; the specification for $V_{\text {slope_inact }}$ for $N a$ conductance is now $-23 \mathrm{mV}$ instead of -7.3 $m V$ (both values are biologically realistic). Fig. 12 represents the diagnosis of re-use for this new set of specifications. It indicates that the specifications of the inactivation sigmoid IP of Sodium current of a Neuron 1 could not be met during the exploration (presence of the red rectangle in the table). At this step, the designer has localized the issue and our tool includes an aid for re-design. The idea is to create a new IP block by resing an existing one.
First of all, the database is re-explored for finding the sigmoid IP that is the closest to the required specifications. This can be performed simply and automatically by minimizing the distance defined by equation (6).

In the current example, the problem comes from the specification of the $V_{\text {slope }}$ parameter. The sizing relation between $V_{\text {slope }}$ and the design parameters, which has been expressed previously in equation (5), can be found in the Documentation file of the sigmoid IP.

To adequately re-size the IP, we just have to change the value of one design parameter, $R$. In our case, we changed the value from $30 \mathrm{k} \Omega$ to $35 \mathrm{k} \Omega$, which had the consequence to shift the $V_{\text {slope }}$ validity domain to include the specification value. The layout view has to be redesigned from the original one. Usually it just consists in enlarging or reducing the size of resistances or capacitors. Nevertheless, in some case, it could be more complex and require the use of automatic layout resizing techniques [25], [50]. This new IP can now be added to the database. Its design time is almost negligible compared to the first-time design of one IP. Thanks to the documentation view, that expresses the relationships between the design parameters and the performance parameters, re-sizing just consists in modifying the design parameters.

\section{CONCLUSION}

Globally, automation of the analog design process is still an open challenge, and re-use methodology is a predominant recommendation of the ITRS. This paper has proposed a synthesis of an experience in analog IC design, centered on the notion of IP. Following the presentation of the issue and review of contributions found in the literature, a methodology is explained in details, with a tutorial intention.

In order to develop an analog design flow based on re-use, some preliminary work is necessary: it includes a clear definition of the system hierarchy, and the identification of the the computation primitives, or IP blocks, at each level of the hierarchy. IP blocks of the lowest level include several transistors and each one is associated with a function. We applied our methodology to the case of neuromimetic ASICs, which design needs can be addressed by our design flow. Furthermore, these devices offer a clear definition of design primitives and system hierarchy.

The starting point of the methodology was an existing library of Hard IP blocks and the first question addressed here is: what information must be added to a Hard IP to transform it into a Firm IP? The proposed answer is (i) to develop a set of models, adapted to multiple abstraction levels, (ii) to formulate the sizing relationships between performance parameters and design parameters and (iii) to characterize to what extent the IP can keep its functionality, when varying the values of performances parameters.

The next point is the implementation of an ASIC design 
flow centered on a database gathering these IPs. A SQL database, including IP packages and hierarchical links between blocks, has been built that allows the implementation of an automatic top-down exploration. We explained the algorithm used to found an IP corresponding to the desired specifications. Finally, the resulting automatic design flow has been tested for two plausible ASIC design projects. These examples show how our web-based platform can assist the designer: it globally accelerates the system design cycle, the designer can quickly see a diagnosis of re-use for his targeted ASIC. In case of total success, the IP packages are immediately retrieved and the designer can perform multi-level simulations of the entire ASIC in a verification phase; in case of partial success, our tool proposes an aid to re-design an IP that matches the specifications.

\section{REFERENCES}

[1] R. Saleh, S. Wilton, S. Mirabbasi, A. Hu, M. Greenstreet, G. Lemieux, P. P. Pande, C. Grecu, and A. Ivanov, "System-on-Chip: Reuse and Integration", Proceedings of the IEEE, vol. 94, $\mathrm{n}^{\circ} .6$, pp. 1050 - 1069, June 2006

[2] M. Keating and P. Bricaud, Reuse methodology manual, 2nd edition ed., Kluwer Academic Publishers, 1999

[3] International Technology Roadmap for Semiconductors (ITRS), Design, Edition 2009

[4] J. Tomas, Y. Bornat, S. Saighi, T. Levi and S. Renaud, "Design of a modular and mixed neuromimetic ASIC", Proceedings ICECS 2006, pp. 946-949, Nice, France, December 2006

[5] T. Levi, N. Lewis, S. Saighi, J. Tomas, Y. Bornat and S. Renaud, "Neuromimetic Integrated Circuits", Chap. 12 in VLSI Circuits for Biomedical Applications, Artech House, Boston, pp. 241-264, 2008

[6] S. Saighi, Y. Bornat, J. Tomas, G. Le Masson and S. Renaud, "A Library of Analog Operators Based on the Hodgkin-Huxley Formalism for the Design of Tunable Real-Time Silicon Neurons", IEEE Transactions on Biomedical Circuits and Systems, vol. 5, pp. 3-19, February 2011

[7] A. Basu, S. Brink, C. Schlottmann, S. Ramakrishnan, C. Petre, S. Koziol, F. Baskaya, C. Twigg and P. Hasler, "A Floating-Gate-Based Field-Programmable Analog Array", IEEE Journal of Solid-State Circuits, vol. 45, n. 9, pp. 1781-1794, September 2010

[8] H. Koh, C. Sequin and P. Gray, "OPASYN: a compiler for CMOS operational amplifiers", IEEE Transactions on Computer-Aided Design of Integrated Circuits and Systems, vol. 9, pp. 113-125, February 1990

[9] R. Phelps, M. Krasnicki, R. Rutenbar, L. Carley and J. Hellums, "Anaconda: simulation-based synthesis of analog circuits via stochastic pattern search", IEEE Transactions on Computer-Aided Design of Integrated Circuits and Systems, vol. 19, pp. 703-717, June 2000

[10] R. Iskander, Knowledge-aware synthesis for analog integrated circuit design and reuse, Dissertation, University Pierre et Marie Curie, Paris VI, July 2008

[11] E. Martens and G. Gielen, High-level modeling and synthesis of analog integrated systems, Springer, January 2008

[12] R. Harjani, R. Rutenbar, and L. R. Carley, "OASYS: A framework for analog circuit synthesis", IEEE Transactions on Computer-Aided Design, vol. 8, no. 12, pp. 1247-1265, December 1989

[13] R. Rutenbar, G. Gielen and J. Roychowdhury, "Hierarchical Modeling, Optimization, and Synthesis for System-Level Analog and RF Designs", Proceedings of the IEEE, vol. 95, n. 3, March 2007

[14] P. Nguyen Tuong, Définition et implantation d'un langage de conception de composants analogiques réutilisables, Dissertation, University Pierre et Marie Curie, Paris VI, June 2006
[15] H. Chang, E. Charbon, U. Choudhury, A. Demir, E. Felt, E. Liu, E. Malavasi, A. Sangiovanni-Vincentelli, and I. Vassiliou, A Top-Down, constraint-Driven Design Methodology for Analog Integrated Circuits, Boston, MA: Kluwer, 1997

[16] G. Gielen, R. Rutenbar, "Computer-aided design of analog and mixed-signal integrated circuits", in Proceedings of the IEEE, vol. 88 , no. 12 , pp. $1825-1852$, December 2000

[17] R. Castro-Lopez, F.V. Fernandez, O. Guerra-Vinuesa and A. Rodriguez-Vasquez, Reuse-Based Methodologies and Tools in the Design of Analog and Mixed-Signal Integrated Circuits, Springer Publishers, 2006

[18] F. De Bernardinis, P. Nuzzo and A.S. Vincentelli, "Robust System Level Design with Analog Platforms", Proceedings Computer-Aided Design ICCAD'06, pp. 334-341, San Jose, US, November 2006

[19] X. Sun, P. Nuzzo, C-C. Wu and A.S. Vincentelli, "Contract-Based System-Level Composition of Analog Circuits", Proceedings ACM/IEEE Design Automation Conference, pp. 605-610, San Francisco, US, July 2009

[20] S. Ohr and L. Marchant, "PANEL : analog intellectual property : now ? or never ?", Proceedings ACM/IEEE Design Automation Conference, pp. 181-182, New Orleans, US, June 2002

[21] R.J. Koch and F. Dielacher, "Analog IP - stairway to SoC heaven?", Proceedings IEEE Solid-State Circuits Conference, pp. 1-2, San Francisco, US, February 2003

[22] R. Singh, "Analog IP re-use: concerns for "digitally-oriented" SoC designers", EETIMES 19/12/2005

[23] R. Goering, "True Circuits rolls out 65-nm analog IP", EETIMES $12 / 06 / 2006$

[24] Z. Li, L. Luo and J. Yuan, "A Study on Analog IP Blocks for MixedSignal SoC", Proceedings ASIC, pp.564-567, Beijing, China, October 2003

[25] M. Dessouky, A. Kaiser, M-M. Louërat and A. Greiner, "Analog Design for Reuse - Case Study : Very Low-voltage $\Delta \Sigma$ Modulator", Proceedings DATE 2000, pp. 353-360, Paris, France, March 2000

[26] B. Gilbert, Design for manufacture, Trade-offs in analog circuit design. The designer's companion, Kluwer Academic Publishers, 2002

[27] VSIA, “Analog/Mixed-Signal VSI Extension Specification Version 2.2”, http://vsi.org/docs/AMS-122-26Feb01.pdf.

[28] J. Michel and F. Schwartz, "Analogue circuit sizing method using interval analysis", Proceedings of the 2008 Joint International IEEE Northeast Workshop on Circuits and Systems and TAISA Conference, NEWCAS-TAISA, pp. 331-334, Montreal, Canada, 2008.

[29] I. O'Connor, F. Tissafi-Drissi, G. Révy and F. Gaffiot, "UML/XMLbased approach to hierarchical AMS synthesis", Proceedings FDL 2005, Lausanne, Switzerland, September 2005

[30] I. O'Connor and al., "CNTFET Modeling and Reconfigurable LogicCircuit Design", IEEE Transactions on Circuits and Systems I, vol. 54, pp. 2365-2379, November 2007

[31] I. Connor and A. Kaiser, "Automated synthesis of current-memory cells", IEEE Transactions on Computer-aided Deign of. Integrated Circuits Systems, vol. 19, no. 4, pp. 413-424, April 2000

[32] R. Castro-Lopez, O. Guerra, E. Roca and F.V. Fernandez, "An Integrated Layout-Synthesis Approach for Analog ICs", IEEE Transactions on Computer-aided Design of Integrated Circuits and Systems, vol. 27, pp. 1179-1189, July 2008

[33] Hamour, M.; Saleh, R.; Mirabbasi and S.; Ivanov, "Analog IP design flow for SoC applications", Proceedings ISCAS 2003, vol. 4, pp. 676-679, Bangkok, Thailand, May 2003

[34] J.V.B. Pimentel and J.C. Da Costa, "A Methodology for Describing Analog/Mixed-Signal Blocks as IP", Design\&Reuse Articles, November 2010

[35] D. M. Binkley, C. E. Hopper, S. D. Tucker, B. C. Moss, J. M. Rochelle and D. P. Foty, "A CAD Methodology for Optimizing Transistor Current and Sizing in Analog CMOS Design", IEEE Transactions on Computer-aided Design of Integrated Circuits and Systems, vol. 22, pp. 225-237, February 2003 
[36] R. Iskander, L. de Lamarre, A. Kaiser and M.-M. Rosset-Louërat, "Design Space Exploration for Analog IPs using CAIRO+", ICEEC International Conference on Electrical Electronic and Computer Engineering , pp. 473-476, Cairo, Egypt, 2004

[37] R. Iskander, M.-M. Louërat and A. Kaiser, "Automatic DC Operating Point Computation and Design Plan Generation for Analog IPs", Analog Integrated Circuit and Signal Processing Journal, vol. 56, 2008

[38] F. Tissafi-Drissi, I. O'Connor, and F. Gaffiot, "RUNE: Platform for automated design of integrated multi-domain systems. Application to high-speed CMOS photoreceiver front-ends", Proceedings of the IEEE Conference on Design Automation and Test in Europe, DATE'04, pp. 16-21, Paris, 2004

[39] A.L. Hodgkin and A.F. Huxley, "A quantitative description of membrane current and its application to conduction and excitation in nerve", Journal of Physiology, 1952

[40] S. Renaud, J. Tomas, N. Lewis, Y. Bornat, A. Daouzli, M. Rudolph, A. Destexhe and S. Saighi, "PAX: A mixed hardware/software simulation platform for spiking neural networks", Journal of Neural Networks, vol. 23, pp. 905-916, April 2010

[41] Verilog-A, Language Reference Manual, Cadence, August 1996

[42] C. J. R. Shi and A. Vachoux, "VHDL-A design objectives and rationale", in Current issues in electronic modeling. vol. 2, K. A. Publishers, pp. 1-30, 1995

[43] F. Pêcheux, C. Lallement and A. Vachoux, "VHDL-AMS and Verilog-AMS as Alternative Hardware Description Languages for Efficient Modeling of Multidiscipline Systems", IEEE Transactions on Computer-Aided Design of Integrated Circuits and Systems, vol. 24, February 2005

[44] R. Bowen, K. Coar, Apache server, Campus Press, 2000

[45] P.Rigaux, Pratique de MySql et Php, O’Reilly, 2006

[46] P. Dubois 2 MySQL, Sams Developer's Library, 2005

[47] S. Abiteboul, P. Buneman, D. Suciu, Data on the web from relations to semistructured data and XML, Morgan Kaufmann Publishers, 2000

[48] T. Levi, J. Tomas, N. Lewis, P. Fouillat, "A CMOS Resizing Methodology for Analog Circuits: linear and non-linear applications", IEEE Journal Design and Test of Computer, vol. 26, pp. 78-87, January-february 2009

[49] S. Renaud, J. Tomas, Y. Bornat, A. Daouzli, S. Saïghi, "Neuromimetic ICs with analog cores: an alternative for simulating spiking neural networks", Proceedings ISCAS 2007, pp 3355-3358, New-Orleans, USA, May 2007

[50] S. Youssef, F. Javid, D. Dupuis, R. Iskander, M. Louerat, “A Pythonbased layout-aware analog design methodology for nanometric technologies", IEEE Design and Test Workshop, pp. 62-67, Beirut, Lebanon, December 2011

Dr. Timothée Levi was born in Talence, France in 1981. He received the Engineer degree in electronics from Electronic Engineering School of Bordeaux (ENSEIRB-MATMECA) and the Master degree in electronics from the University of Bordeaux 1, France in 2004. He received the Ph.D. degree in Electrical, from the University of Bordeaux 1 in 2007. His dissertation was about the reuse methodology applied on neuromorphic engineering.

In 2008, he was a post-doctoral researcher at CEA-LETI in Grenoble, France, about real-time signal processing of spike sorting. In 2009, he was post-doctoral researcher at University of Tokyo (LIMMS / IIS), Japan, about silicon neural network for smart MEMS systems.

$\mathrm{He}$ is currently Associate Professor at Laboratory IMS (CNRS/ENSEIRB, University Bordeaux 1) since 2010. His main research is focused on architecture of silicon neural networks and reuse methodologies. He authored and co-authored 2 chapters of book, 6 journal articles, one patent and about 20 conference articles.

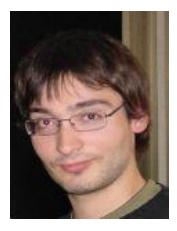

Dr. Noëlle Lewis holds a Ph.D. in Electronics from Bordeaux University, France, in 1997 and she is associate professor since 2000 at IMS laboratory, at Bordeaux University. Her first topic of interest was behavioral modeling of analogue integrated circuits. She joined the "Engineering of Neuromorphic Systems" (ISN) research team of IMS lab in 2005, to contribute to solve some critical points related to analogue IC integration with IP re-use techniques. Since 2010, she is the head of a new research team focusing on Electronic Systems Interacting with Biology, ELIBIO, a research team in which 3 faculties, $3 \mathrm{Ph}$. D. students and 2 engineers are involved. Currently, this team participates to 3 projects funded by the french National Research Agency and covering new fields of specializations like: biomedical instrumentation, low power low frequency IC for bio-signal recording, real-time low power signal processing and closed-loop neural stimulation.

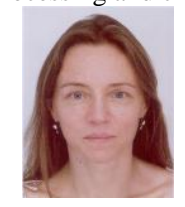

Dr. Jean Tomas received the M.Sc. degree in Electrical Engineering from Ecole Nationale Supérieure d'Electronique et de Radioélectricité de Bordeaux (ENSERB) in 1985 and the Ph.D. degree in Electrical Engineering from Université Bordeaux 1 in 1988.

His research interests include design of analog and mixed signal circuits and systems dedicated to neuromorphic applications. He has also authored and co-authored more than 50 peer-review publications.

Currently, he is Associate Professor at Université Bordeaux 1 in IMS Laboratory (UMR 5218 CNRS / Université de Bordeaux).

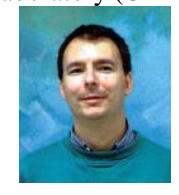

Pr. Sylvie Renaud graduated in electronic engineering (MSc) in Supelec (Paris-France) in 1986. She received her PhD in Physics at the University of Bordeaux (France) in 1990, and her HDR (Research Habilitation) in 2001. After a post-doctoral stay at Brandeis University (MA, USA) in 1991_1992, she was appointed as an Assistant-Professor, then Professor in ENSEIRB Bordeaux (National Engineering School) where she was recently appointed as Research Board Director in the Institut Polytechnique de Bordeaux.

She created in 1994 the « Engineering of Neuromorphic Systems » group in IMS-Labs (Univ. Bordeaux, CNRS, ENSEIRB), and now heads the BioElectronics group with 12 permanent researchers. Her research interests are: analog and mixed neuromorphic VLSI; real-time hardware simulation platforms of spiking neural networks; hybrid systems interfacing living and artificial neurons; analog ASICs for biological signal conditioning and events detection; active VLSI implants for neurodegenerative diseases and diabetes; closed-loop living-artificial systems. She authored and co-authored more than 50 reviewed international articles and communications.

Prof Renaud is an expert for the EU commission on FET and ICT calls, and for NS-NIH on CRCNS calls. She is a reviewer for IEEE journals and conferences since 1997 and organizes special Sessions, Tutorials and workshops in IEEE conferences on a regular basis.

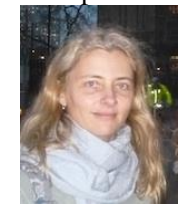

